\title{
Seven-Year Follow-up of PCL Reconstruction Combined with Opening-Wedge High Tibial Osteotomy: A Case Report
}

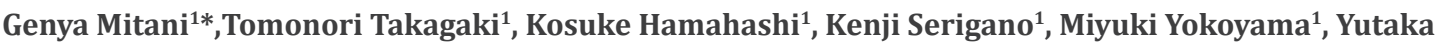 \\ Nakamura $^{2}$, Masato Sato ${ }^{1}$, Masahiko Watanabe ${ }^{1}$ \\ 1* Department of Orthopaedic Surgery, Tokai University Oiso Hospital Gakkyou, Oiso, Naka-gun, Kanagawa, Japan \\ ${ }^{2}$ Department of Physical Recreation, School of Physical Education, Tokai University, Kitakaname, hiratsuka, Kanagawa, Japan
}

Received: June 15, 2016; Accepted: July 29, 2016; Published: August 30, 2016

*Corresponding author: Genya Mitani MD, phD,Assistant Professor,Depatment of Orthopaedic Surgery, Tokai University Oiso Hospital,Address: Gakkyou 21-1, Oiso, Naka-gun, Kanagawa 259-0198,Japan.Tel: +81(463)72-3211;Fax: +81(463)72-2256;E-Mail: genya@syd.odn.ne.jp

\begin{abstract}
The treatment of progressive osteoarthritis $(\mathrm{OA})$ secondary to cruciate ligament insufficiency in middle-aged patients is of concern. We present a case report ofan active 48-year-oldman with progressive knee $\mathrm{OA}$ secondary to posterior cruciate ligament (PCL) and posterolateral corner (PLC) deficiency. Physical examination revealed a positive posterior drawer sign, tibia posterior sagging sign, and dial test with severe varus deformityof the left knee. Plain radiographs of the left knee showed medial joint space narrowing, $186^{\circ}$ femorotibial angle (FTA), and $0 \%$ weight-bearing line (WBL) percentage. Magnetic resonance imaging showed extended arthritic changes in the medial compartment and apparent disappearance of the PCL. We performed PCL reconstruction using the hamstring tendons combined with an opening-wedge high tibial osteotomy (OWHTO) and followed the patient for 7 years. Knee function improved satisfactorily and there was a negative posterior drawer sign. The clinical scores improved after the operation. The clinical scores before, 2 years after, and 7 years after the operation were as follows: Lysholm score: 40, 91, and 90; International Knee Documentation Committee score: 37.9, 73.0, and 72.4; and Knee Society Score: 50.0, 91.2, and 85.0.The acquired stability and function of the knee had been maintained for 7 years after the operation. Follow-up plain radiographs at 7 years showed recovery of the arthritis changes in the medial joint space, no progression of arthritis in the lateral joint space, and maintenance of the corrected angle (65\% WBL percentage, $169^{\circ} \mathrm{FTA}$ ).We conclude that PCL reconstruction combined with OWHTO is a feasible option in such patients.
\end{abstract}

Keywords: Posterior cruciate ligament reconstruction; posterolateral corner; opening-wedge high tibial osteotomy

\section{Introduction}

The surgical techniques, rehabilitation, and clinical results of ligament reconstruction have improved in recent years. As a result, the indications for posterior cruciate ligament (PCL) reconstruction, which was managed conservatively before such improvements, have increased. This increase in indications may be applicable to middle-aged and older patients with osteoarthritis(OA) arising from a previous cruciate ligament injury who would have been considered for arthroplasty in the past. This case report describes a 7-year follow-up of a patient who underwent PCL reconstruction combined with an openingwedge high tibial osteotomy (OWHTO) for progressive knee OA caused by a previous PCL injury and posterolateral corner (PLC) deficiency.

\section{Case Report}

A 48-year old man came to our outpatient clinic with complaints of severe pain and instability in his left knee. He had sustained a left knee sprain at the age of 22 years. Although he continued to feel knee instability after recovery, this was left unsolved, and the knee pain had increased gradually with age. There were no appreciable past medical and family histories.

On physical examination, the left knee exhibited effusion and severe varus deformity with muscle atrophy of the left thigh and lower leg. The knee had a positive posterior drawer sign, tibiaposterior sagging sign, and dial test with a range of motion of $5^{\circ}$ to $130^{\circ}$. The Lysholm score was 40 , the International Knee Documentation Committee (IKDC) score was 37.9, and the Knee Society Score (KSS) was 50.

A plain anteroposterior (AP)radiograph showed some spur formation and joint space narrowing of the medial femorotibial joint of the left knee(Figure $1 \mathrm{~A}$ ).The tibialslope was $7.4^{\circ}$ in a plain lateral radiograph (Figure 1B). The femorotibial angle (FTA) was $186^{\circ}$, and the weight-bearing line (WBL) percentage (1) was $0 \%$ as measured in standing long-cassette radiographs of the lower extremities (Figure1C). Magnetic resonance imaging (MRI) showed extended arthritic changes in the medial compartment (Figure 2A) and apparent disappearance of the PCL (Figure 2B, 2C).

We diagnosed this as knee osteoarthritis secondary to PCL and PLC deficiency. We performed a PCL reconstruction combined with an HTO 26 years after the initial knee injury. Intra operative arthroscopic findings showed a wide spectrum 
of International Cartilage Repair Society (ICRS) grade 4 cartilage damage and disappearance of the PCL (Figure 3A). Although there was no cartilage damage or meniscus injury in the lateral joint space, a positive gap sign (enlargement of the lateral joint space) was observed in the crossed-leg position (Figure 3B).

After arthroscopic synovectomy and spur resection, we performed the OWHTO. The surgery was performed according to the method reported in Takeuchi et al(1). The correction angle was set at $16^{\circ}$ to give aWBL percentage of $65 \%$. $\beta$-Tricalcium phosphate (porosity 60\%; Olympus Biomaterial, Tokyo, Japan) was used to fill the opening space of the osteotomy, and a Tomo FixMedial High Tibial Plate (Johnson and Johnson DePuy Synthes Trauma, New Brunswick, NJ, USA) was used as the fixation device. An anatomical bisocket PCL reconstruction using a semitendinosus and gracilis tendon auto graft was performed. The proximal end of the graft was secured with an Endo Button CL fixation device, and the distal end of the graft was fixed on the tibia using a Double-Spike Plate system (Smith \& Nephew, Andover, MA, USA) (Figure 4). The knee was immobilized with a plaster cast.

Postoperative rehabilitation was begun 2 weeks later and comprised passive range-of-motion exercises using continuous passive motion and muscle strengthening. Standing exercises were also initiated as soon as possible. The patient began partial weight-bearing exercises 4 weeks after the operation and full weight-bearing walking 6 weeks after the operation.

Second-look arthroscopy was performed at the time of removal of the implants at 1 year after the operation. This showed excellent tension and structure of the reconstructed PCL (Figure 5A), and cartilage resurfacing of the medial femorotibial joint surface (Figure 5B).
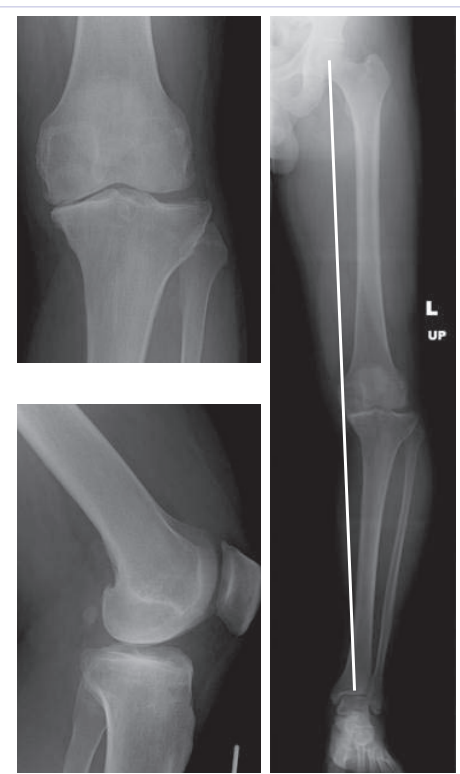

Figure 1: A: A plain AP radiograph showed some spur formation and joint space narrowing in the medial femorotibial joint of the left knee.B:The tibial slope was $7.4^{\circ}$ in a plain lateral radiograph. C: The FTA was $186^{\circ}$ and the WBL percentage was $0 \%$ as measured in standing long-cassette radiographs of the lower extremities..

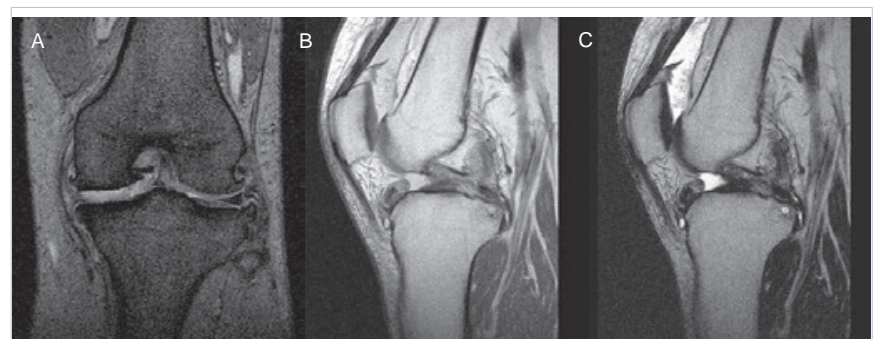

Figure 2: Preoperative MRI of the left knee. A: T2 weighted coronalMRIslice depicting extended arthritic changes in the medial compartment. B and C: T1 and T2 weighted sagittal MRI slice depicting disappearance of the PCL.
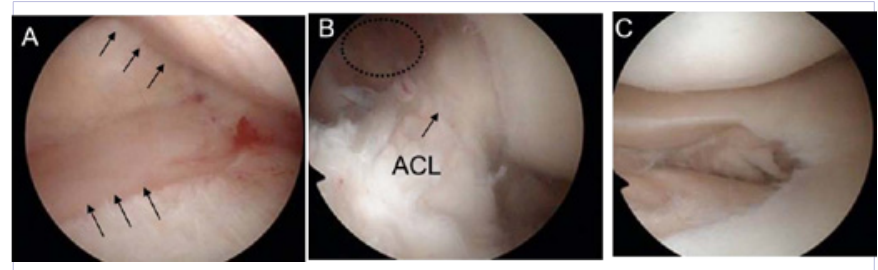

Figure 3: meniscus injury in the lateral joint space, a positive gap sign (enlargement of the lateral joint spacev)was seen in the crossed-leg position.

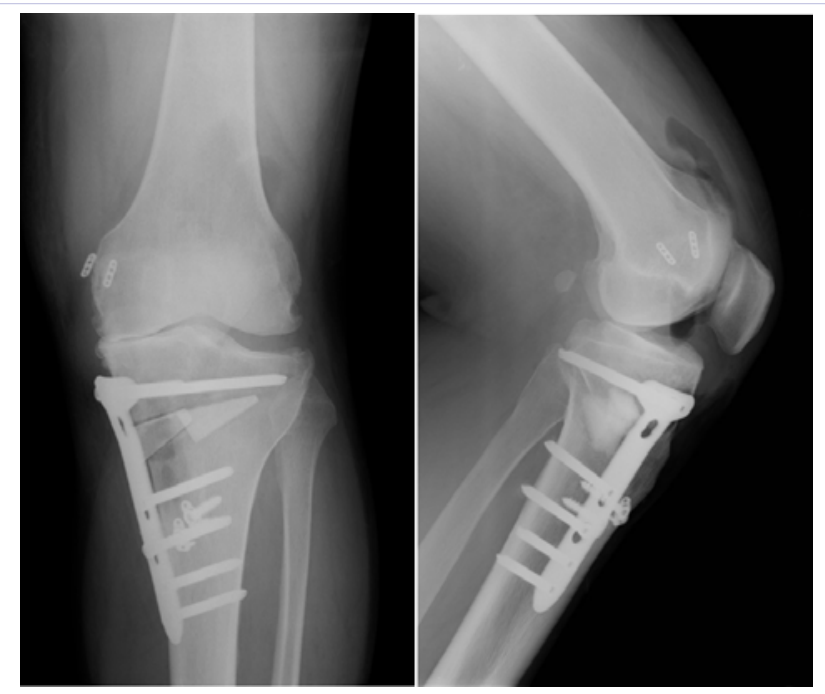

Figure 4: Postoperative plain radiographs showing the results of the PCL reconstruction combined with OWHTO.

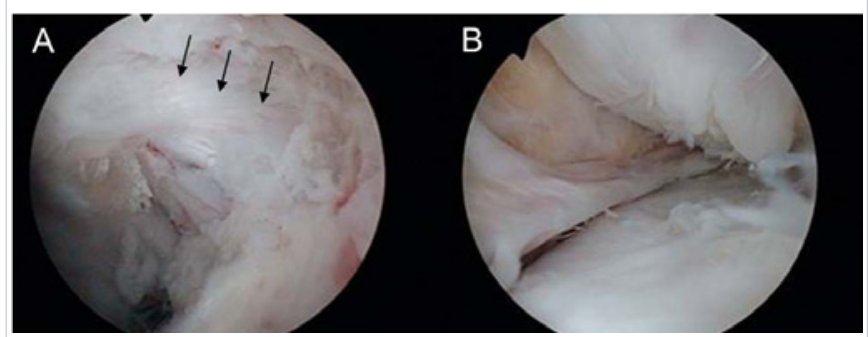

Figure 5: A: Second-look arthroscopy showed excellent tension and structure of the reconstructed PCL.B: Cartilage resurfacing of the medial femorotibial joint surface. 
Plain AP radiograph 7 years after the operation showed recovery of the arthritis changes in the medial joint space, no progression of arthritis in the lateral joint space (Figure 6A). The tibial slope was $12.7^{\circ}$ in a plain lateral radiograph (Figure $6 \mathrm{~B})$. The FTA was $169^{\circ}$ and the WBL percentage was $65 \%$ as measured in standing long-cassette radiographs of the lower extremities (Figure 6C).

The postoperative clinical scores for before, 2 years after, and 7 years after the operation were as follows: Lysholm score:40, 91, and 90; IKDC score: 37.9, 73.0, and 72.4; and KSS: 50.0, 91.2, and 85.0. The stability and function of the knee had been maintained for up to 7 years after the operation.

\section{Discussion}

Until recently, PCL injury has tended to be treated conservatively because of the difficulties in surgical techniques, the risk of graft looseness caused by a "killer turn" (2), and acceptable results of conservative treatment. Patel et al. reported that most patients with an acute, isolated PCL injury do well with non operative treatment at an intermediate-term followup (3).However, older patients experiencing symptoms caused by instability or multi ligament injury(especially associated with PLC deficiency)should be considered as operative indications.

PLC affects the lateral collateral ligament, popliteus tendon, popliteofibular ligament, arcuate ligament, and fabellofibular ligament.PLC is usually damaged in combination with rupture of one of the cruciate ligaments by direct or indirect trauma to the knee. Injury to the posterolateral corner is often missed but

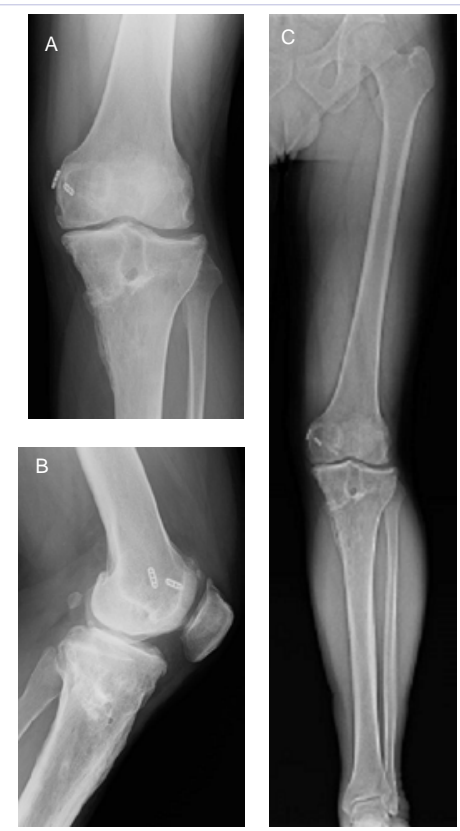

Figure 6: A: Plain AP radiograph at the 7-year follow-up showed recovery of the arthritis changes in the medial joint space, no progression of arthritis in the lateral joint space. B: The tibial slope was $12.7^{\circ}$ in a plain lateral radiograph. C: The FTA was $169^{\circ}$ and the WBL percentage was $65 \%$ as measured in standing long-cassette radiographs of the lower extremities. Correction angle has maintained for 7 years. can contribute greatly to knee instability and is a potential factor in the failure of cruciate ligament reconstruction (4).Some of the reasons that these structures can be difficult to image even when using coronaloblique images of MRI(5,6).

In a cadaveric study of posterolateral rotatory instability of the knee joint, Kaneda et al. reported that sectioning of the PCL alone did not increase the amount of external tibial rotation, but cutting the PCL after sectioning of the PLC increased external tibial rotation(7).

Although the non operative treatment of PLC injuries tends to yield poor results, the results of reconstruction surgery for old PLC injuries are not satisfactory. In patients with chronicPLC injury, failure to correct a genu varus alignment will often result in failure of the PLC repair or reconstruction. OWHTO is now used for treatment of genu varus alignment because of its advantages in maintaining the bone stock, its ability to correct deformities close to their origin, and the lack of a need for a fibula osteotomy. Additionally, development of internal fixation device sallow's for the prevention of possible loss of correction and more rigid fixation for early weight bearing (1).

Several authors have reported increased tibialslope and negative effects of OWHTO (8-10). By contrast, Arthur et al. reported that OWHTO can be an effective first method of treatment for patients with chronic combined posterolateral knee injuries and genu varus alignment (11). Savarese et al. reported that chronic ligament deficiency and malalignment are good indications for HTO because it can change the coronal as well as the sagittal plane of the knee. Increased tibial slope causes anterior tibia translation and helps the PCL-deficient knee (12).

There are conflicting opinions about using simultaneous or staged surgery for such cases. Even in the report that recommends OWHTO as the initial treatment by Arthur et al. (11), most of patients with a high-velocity or multiligament injury required second-stage ligament reconstruction. Additionally, although simultaneous HTO and ligament reconstruction surgery istechnically demanding, we have sufficient technical experience in performing anterior cruciate ligament reconstruction combined with HTO proactively. For these reasons and considering that this case involved medial side $\mathrm{OA}$ with genu varus secondary to old PCL and PLC injuries in an active middle-aged patient and OWHTO is also an option for treating PLC insufficiency, we performed PCL reconstruction combined with HTO without PLC reconstruction. The clinical results and radiographic examination showed improvements over the short term and that these had been maintained at the 7-year follow-up. Therefore, we conclude that PCL reconstruction combined with OWHTO is one option for patients with medial side OA secondary to insufficiency of the PCL and PLC.

\section{Abbreviations}

PCL: posterior cruciate ligament, OA: osteoarthritis, OWHTO: opening-wedge high tibial osteotomy, PLC: posterolateral corner,IKDC: International Knee Documentation Committee, KSS: Knee Society Score, AP: anteroposterior, AP: anteroposterior, FTA: femorotibial angle, WBL: weight-bearing line, MRI: magnetic resonance imaging, ICRS: International Cartilage Repair Society 


\section{Acknowledgments}

The authors acknowledge Dr. Taisuke Tomatsu for great mentorship in the performance of the knee surgery.

\section{Declarations}

The authors declare that they have no competing interests.

\section{References}

1. Takeuchi R, Ishikawa H, Aratake M, Bito H, Saito I, Kumagai K, et al. Medial opening wedge high tibial osteotomy with early full weight bearing. Arthroscopy. 2008;25(1):46-53. doi: 10.1016/j arthro.2008.08.015.

2. Weimann A, Wolfert A, Zantop T, Eggers AK, Raschke M, Petersen W. Reducing the "killer turn" in posterior cruciate ligament reconstruction by fixation level and smoothing the tibial aperture. Arthroscopy. 2007;23(10):1104-1111.

3. Patel DV, Allen AA, Warren RF, Wickiewicz TL, Simonian PT. The nonoperative treatment of acute,isolated(partial or complete) posterior cruciate ligament-deficient knees: an intermediate-term follow-up study.HSS J. 2007;3(2):137-146. doi: 10.1007/s11420007-9058-z.

4. Davies H, Unwin A, Aichroth P. The posterolateral corner of the knee anatomy, biomechanics and management of injuries. Injury, Int. J. Care Injured. 2004;35:68-75.

5. Yu JS, Salonen DC, Hodler J, Haghighi P, Trudell D, Resnick D Posterolateral aspect of the knee:improved MR imaging with a coronal oblique technique. Radiology. 1996;198(1):199-204.
6. LaPrade RF, Gilbert TJ, Bollom TS, Wentorf F, Chaljub G. The magnetic resonance imaging appearance of individual structures of the posterolateral knee. A prospective study of normal knees and knees with surgically verified grade III injuries. Am J Sports Med. 2000;28(2):191-199.

7. Kaneda Y, Moriya H, Takahashi K, Shimada Y, Tamaki T.Experimental study on external tibial rotation of the knee. AmJSports Med. 1997;25(6):796-800.

8. Noyes FR, Goebel SX, West J. Opening wedge tibial osteotomy: the 3-triangle method to correct axial alignment and tibial slope. Am J Sports Med. 2005;33(3):378-387.

9. Rodner CM, Adams DJ, Diaz-Doran V, Tate JP, Santangelo SA, et al. Medical opening wedge tibial osteotomy and the sagittal plane: the effect of increasing tibial slope on tibiofemoral contact pressure. Am J Sports Med. 2006;34(9):1431-1444.

10. Marti CB, Gautier E, Wachtl SW, Jakob RP. Accuracy of frontal and sagittal plane correction in open-wedge high tibial osteotomy. Arthroscopy.2004;20(4):366-372.

11. Arthur A, LaPrade RF, Agel J. Proximal tibial opening wedge osteotomy as the initial treatment for chronic posterolateral corner deficiency in the varus knee.Am J Sports Med. 2007;35(11):1844-1850. Epub 2007 Aug 27.

12. Savarese E, Bisicchia S, Romeo R, Amendola A. Role of high tibial osteotomy in chronic injuries of posterior cruciate ligament and posterolateral corner. J Orthop Traumatol. 2011;12(1):1-17. doi: 10.1007/s10195-010-0120-0. 